3. Dokun AO, Kim S, Smith HR, et al. Specific and nonspecific NK cell activation during virus infection. Nat Immunol 2001 ; 2 : 951-6.

4. Robbins SH, Tessmer MS, Mikayama T, Brossay L. Expansion and contraction of the NK cell compartment in response to murine cytomegalovirus infection. J Immunol 2004 ; 173 : 259-66.

5. Bekiaris V, Timoshenko 0 , Hou TZ, et al. Ly49H NK cells migrate to and protect splenic white pulp stroma from murine cytomegalovirus infection. J Immunol 2008; $180: 6768-76$.
6. O'Leary JG, Goodarzi M, Drayton DL, von Andrian UH $T$ cell- and $B$ cell-independent adaptive immunity mediated by natural killer cells. Nat Immunol 2006; $7: 507-16$.

7. Cooper MA, Elliott JM, Keyel PA, et al. Cytokineinduced memory-like natural killer cells. Proc Natl Acad Sci USA 2009; 106 : 1915-9.

8. Arstila TP, Casrouge A, Baron V, et al. Diversity of human alpha beta T cell receptors. Science 2000 ; 288: 1135 .

9. Badovinac VP, Haring JS, Harty JT. Initial T cell receptor transgenic cell precursor frequency dictates critical aspects of the $\mathrm{CD}^{+} \mathrm{T}$ cell response to infection. Immunity 2007; 26: 827-41.

10. Kaech SM, Tan JT, Wherry $\varepsilon$ J, et al. Selective expression of the interleukin 7 receptor identifies effector CD8 $T$ cells that give rise to long-lived memory cells. Nat Immunol 2003; 4 : 1191-8.

11. Almeida JR, Price DA, Papagno L, et al. Superior control of HIV-1 replication by $\operatorname{CD} 8^{+} T$ cells is reflected by their avidity, polyfunctionality, and clonal turnover. J Exp Med 2007 ; 204 : 2473-85.

\title{
NOUVELLE
}

\section{La clé vers de nouveaux inhibiteurs de iNOS}

Elsa Garcin
University of Maryland Baltimore County, Department of Chemistry and Biochemistry, 1000 Hilltop Circle, 21250 Baltimore, États-Unis. egarcin@umbc.edu
> Contrôler l'activité d'une enzyme sans perturber l'activité d'enzymes de la même famille est l'un des plus grands défis auxquels est confrontée la découverte de nouveaux traitements anti-inflammatoires, anticancéreux et bien d'autres; le risque est en effet la survenue d'effets secondaires indésirables. C'est par exemple le cas des anti-inflammatoires non stéroïdiens qui bloquent la production de prostaglandines, molécules partiellement responsables de l'inflammation et produites par deux enzymes de la même famille (les cyclooxygénases Cox-1 et Cox-2). Les traitements classiques ne faisant pas la différence entre Cox-1 et Cox-2, ils exposaient les patients à des effets indésirables touchant le système digestif (brûlures d'estomac, ulcères, perforations et hémorragies gastriques). La deuxième génération d'anti-inflammatoires (Coxib), qui a une très grande spécificité pour Cox2 par rapport à Cox-1, entraîne moins de troubles digestifs [1]. Nos études récentes se sont concentrées sur une enzyme, la Nitric oxide synthase (NOS), associée à de nombreuses maladies allant de l'inflammation au cancer, et elles suggèrent de nouvelles stratégies pour tenter de résoudre ce problème [2].

Jusque dans les années 1980, le monoxyde d'azote (NO, nitric oxide), produit à partir d'oxygène et de l'acide aminé L-arginine par l'enzyme Nitric Oxide Synthase (NOS), était considéré comme nocif et polluant. Dix ans plus tard, F. Murad, L.J. Ignarro, R. Furchgott et S. Moncada [3-6] démontrent que ce gaz est aussi responsable de la relaxation vasculaire dans l'organisme. NO joue en fait un double rôle: celui de messager dans les systèmes cardiovasculaire et nerveux, et celui d'intermédiaire de la réponse immunitaire.

\section{Les trois formes}

\section{de nitric oxide synthase}

Dans l'organisme, l'enzyme NOS existe sous trois formes : la forme neuronale (nNOS), la forme induite (iNOS), et la forme endothéliale (eNOS). Les enzymes endothéliale et neuronale sont exprimées de façon constitutive et leur activité est contrôlée entre autres par la concentration en calcium dans la cellule. Ces deux enzymes produisent peu de NO, mais en quantité suffisante pour d'une part, réguler la pression sanguine et éviter l'agrégation plaquettaire via l'activation de l'enzyme soluble guanylate cyclase (sGC), d'autre part, permettre la transmission d'information entre cellules nerveuses façonnant nos fonctions de mémorisation et d'apprentissage. La forme induite iNOS est, quant à elle, principalement exprimée dans les macro- phages exposés à une agression bactérienne, virale ou tumorale, et réagissant par l'intermédiaire de molécules incluant les lipopolysaccharides bactériens (LPS), les cytokines (interféron- $\gamma$ ), et le facteur nécrosant des tumeurs (tumor necrosis factor, TNF- $\alpha$ ). iNOS est également exprimée dans d'autres types de cellules, dont les neutrophiles, les cellules hépatiques, les chondrocytes composant le cartilage, et les fibres musculaires. De surcroît, son activité est très peu régulée. Produisant de larges quantités de NO sur demande, iNOS est l'enzyme idéale dans le cadre de la réponse immunitaire. Cependant, cette hyperactivité de iNOS a un revers: un surplus de NO peut induire une dilatation trop importante des vaisseaux sanguins à l'origine par exemple d'hypotension dans le cas du choc septique. De nombreuses autres manifestations pathologiques sont associées à la surproduction de NO par iNOS: inflammation, arthrite, rejet de greffe, et cancer; au contraire, une production de NO insuffisante est associée à l'artériosclérose et à l'hypertension artérielle [7]. Il est donc important de pouvoir contrôler précisément la production de NO par iNOS sans pour autant diminuer les activités des autres enzymes eNOS et nNOS nécessaires au fonctionnement des systèmes vasculaire et nerveux. 


\section{Comment créer}

des inhibiteurs spécifiques de iNOS

Pour réduire l'activité de iNOS, une stratégie consiste à utiliser des molécules qui se fixent dans le site actif de l'enzyme où intervient la formation de NO. Ces molécules ou inhibiteurs entrent en compétition avec le substrat L-arginine et empêchent la réaction catalysée par iNOS. II existe plus de 100 inhibiteurs de NOS avec des affinités variables pour chaque enzyme [8]. Une des plus grosses difficultés que rencontre le développement d'inhibiteurs de iNOS est que ces molécules doivent être non seulement très actives contre iNOS, mais aussi très peu actives contre eNOS et nNOS afin de préserver la fonction de messager de NO dans la cellule. Or des études structurales ont montré que les sites actifs des trois enzymes sont quasiment identiques [913]. Pour tenter de résoudre ce problème, nous avons utilisé la cristallographie aux rayons $X$. Cette technique permet de visualiser la structure 3D des protéines (Figure 1A), d'identifier les positions des atomes composant les protéines, et de déterminer avec précision le mode de fixation des inhibiteurs dans le site actif de ces protéines. Nous avons étudié 14 inhibiteurs provenant de deux familles distinctes de molécules, ou pharmacophores, en utilisant iNOS et eNOS comme cibles [2] (Figure 1B). En comparant les structures 3D de différents inhibiteurs fixés dans iNOS et eNOS, nous avons pu identifier des modes de fixation caractéristiques de chacune des formes de NOS. Nous avons montré que ces inhibiteurs non seulement sont ancrés dans le site actif grâce à des liaisons hydrogènes, des interactions hydrophobes et des ponts

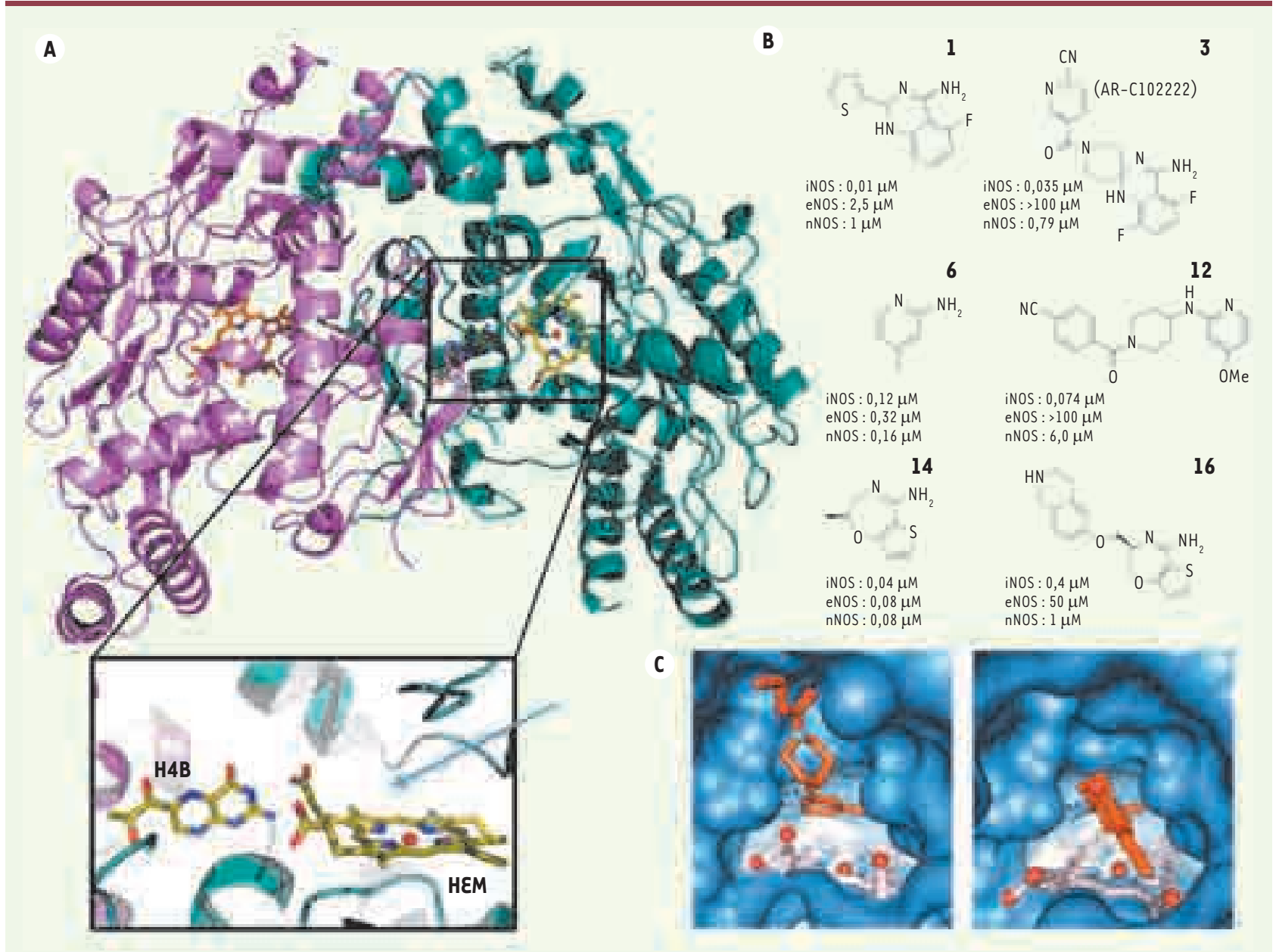

Figure 1. A. Structure 3D de la NOS dimérique représentée par un ruban reliant les atomes $C \alpha$ de chaque acide aminé formant la protéine. Les deux chaînes polypeptidiques sont colorées en magenta et vert. Les cofacteurs hème (HEM) et tétrahydrobioptérine (H4B) représentés sous forme de bâtons, forment le site actif de la protéine où a lieu la réaction. Le site de fixation des inhibiteurs est indiqué par la flèche dans la partie agrandie qui montre uniquement le site actif de NOS. B. Structure de 6 inhibiteurs représentatifs de trois classes différentes ( 1 et $3:$ quinazolines; 6 et 12 : aminopyridines; 14 et 16 : bicyclic thioenooxazepines). Les constantes d'inhibition (IC50: concentration d'inhibiteur nécessaire pour réduire l'activité de l'enzyme de $50 \%$ ) sont indiquées pour chaque forme de NOS. C. Vue du mode de fixation d'un inhibiteur (orange) au dessus de l'hème (gris) dans le site actif de NOS montrant l'ouverture de la nouvelle cavité dans iNOS (gauche) mais pas dans eNOS (droite). 
salins, mais qu'ils s'accrochent également à l'extérieur du site actif, via des liaisons hydrogènes et des interactions hydrophobes. Étant donné que les sites actifs des deux enzymes sont identiques, les modes de fixation de ces inhibiteurs dans leurs sites actifs sont également très semblables. En revanche, leur mode de fixation à l'extérieur du site actif est très différent en ce qui concerne iNOS et eNOS.

Nous avons montré grâce à des études de mutagenèse couplées à des tests d'activité in vitro et in vivo et à des études cristallographiques que la fixation externe des inhibiteurs est due à une cascade de mouvements d'acides aminés commençant dans le site actif et aboutissant à l'ouverture d'une nouvelle cavité dans iNOS (Figure 1C). Dans eNOS, l'ouverture de cette cavité est impossible en raison de la présence d'un acide aminé plus gros que dans iNOS, et bloquant la cascade de mouvements à l'extérieur du site actif (Figure 1C). Cet acide aminé (aa) fait partie d'un trio d'acides aminés spécifique à chaque forme de NOS; ces trois acides aminés sont essentiels et déterminent en grande partie la spécificité de ces inhibiteurs pour chaque enzyme. On peut faire un parallèle avec ce qui se passe dans un jeu de dominos: dans le cas de iNOS, la chute d'un premier domino - provoquée par la fixation de l'inhibiteur dans le site actif - induit la chute d'autres dominos et aboutit à l'ouverture d'une nouvelle cavité permettant à l'inhibiteur de se fixer à l'extérieur du site actif. Dans eNOS, le jeu est bloqué : un acide aminé qui est plus gros que dans iNOS empêche la cascade de chutes de dominos et l'ouverture de cette nouvelle cavité. Nos résultats montrent donc qu'un trio d'acides aminés situés loin du site actif explique pourquoi un inhibiteur très spécifique pour une forme de NOS (iNOS) sera complètement inactif pour l'autre enzyme (eNOS).

Les résultats décrits ci-dessus nous ont permis de développer une nouvelle approche de conception d'inhibiteurs spécifiques de iNOS. Ces inhibiteurs contiennent un module de base qui leur permet de se fixer de façon efficace au site actif des NOS. Des groupements variés sont attachés au module de base de façon à provoquer une cascade de mouvements subtils dans la protéine et promouvoir l'ouverture de nouvelles cavités à l'extérieur du site actif. En interagissant avec des parties de la protéine qui sont situées loin du site actif et présentent un faible degré de similitude entre les différentes formes d'enzymes, ces groupements permettent de contourner le principal obstacle que représente la très grande similitude des sites actifs des différentes formes de NOS. Nous avons validé cette approche en créant de nouveaux inhibiteurs spécifiques de iNOS: au module de base qui assure une fixation de l'inhibiteur dans le site actif, nous ajoutons progressivement des groupements de plus en plus gros pour déclencher la cascade de mouvements conduisant à l'ouverture d'une nouvelle cavité dans iNOS.

\section{Une méthode généralisable?}

Des stratégies similaires pourraient peutêtre être appliquées à la création d'inhibiteurs d'autres familles d'enzymes, comme les kinases, la transcriptase inverse du virus d'immunodéficience humaine (VIH), ou encore les cyclo-oxygénases (COX). Ces dernières partagent également un degré de similitude élevé de leur site actif, ce qui rend difficile la conception d'inhibiteurs spécifiques, pourtant nécessaires pour éviter des effets secondaires négatifs. $\diamond$ A novel strategy to design specific iNOS inhibitors

\section{RÉFÉRENCES}

1. Farooq M, Haq I, Qureshi AS. Cardiovascular risks of COX inhibition: current perspectives. Exp Opin Pharmacother $2008 ; 9$ : 1311-9.

2. Garcin ED, Arvai AS, Rosenfeld RJ, et al. Anchored plasticity opens doors for selective inhibitor design in NOS. Nat Chem Biol 2008; 4 : 700-7

3. Palmer RMJ, Ferrige AG, Moncada S. Nitric oxide release accounts for the biological activity of endothelium-derived relaxing factor. Nature 1987 ; 327 : 524-6.

4. Ignarro LJ, Buga GM, Wood KS, et al. Endothelium-derived relaxing factor produced and released from artery and vein is nitric oxide. Proc Natl Acad Sci USA 1987 ; 84 : 9265-9.

5. Khan MT, Furchgott R. Additional evidence that endothelium-derived relaxing factor is nitric oxide. Amsterdam : Elsevier, 1987

6. Katsuki S, Arnold W, Mittal C, et al. Stimulation of guanylate cyclase by sodium nitroprusside, nitroglycerin and nitric oxide in various tissue preparations and comparison to the effects of sodium azide and hydroxylamine. J Cyclic Nucleotide Res 1977 ; 3 : 23-35.

7. Bian K, Murad F. Nitric oxide (NO): biogeneration, regulation, and relevance to human diseases. Front Biosci $2003 ; 8: \mathrm{d} 264-78$.

8. Tafi A, Angeli L, Venturini G, et al. Computational studies of competitive inhibitors of nitric oxide synthase (NOS) enzymes: towards the development of powerful and isoform-selective inhibitors. Curr Med Chem 2006; $13:$ 1929-46.

9. Crane BR, Arvai AS, Ghosh DK, et al. Structure of nitric oxide synthase oxygenase dimer with pterin and substrate. Science $1998 ; 279$ : 2121-6.

10. Fischmann TO, Hruza A, Niu XD, et al. Structural characterization of nitric oxide synthase isoforms reveals striking active-site conservation. Nat Struct Biol 1999 ; $6: 233-42$.

11. Raman CS, Li H, Martasek P, et al. Crystal structure of constitutive endothelial nitric oxide synthase: a paradigm for pterin function involving a novel metal center. Cell $1998 ; 95$ : 939-50.

12. Flinspach M, Li H, Jamal J, et al. Structures of the neuronal and endothelial nitric oxide synthase heme domain with D-nitroarginine-containing dipeptide inhibitors bound. Biochemistry $2004 ; 43$ : 5181-7.

13. Fedorov R, Hartmann $\varepsilon$, Ghosh DK, et al. Structural basis for the specificity of the nitric-oxide synthase inhibitors W1400 and N(omega)-propyl-L-arg for the inducible and neuronal isoforms. J Biol Chem 2003; $278: 45818-25$.

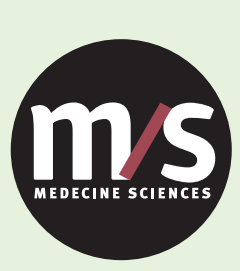

Tarifs d'abonnement M/S - 2009

$>$ Grâce à $m / s$, vous vivez en direct les progrès des sciences biologiques et médicales

Abonnez-vous

à Médecine/Sciences

\section{Bulletin d'abonnement page 568 dans ce numéro de $\mathrm{m} / \mathrm{s}$}
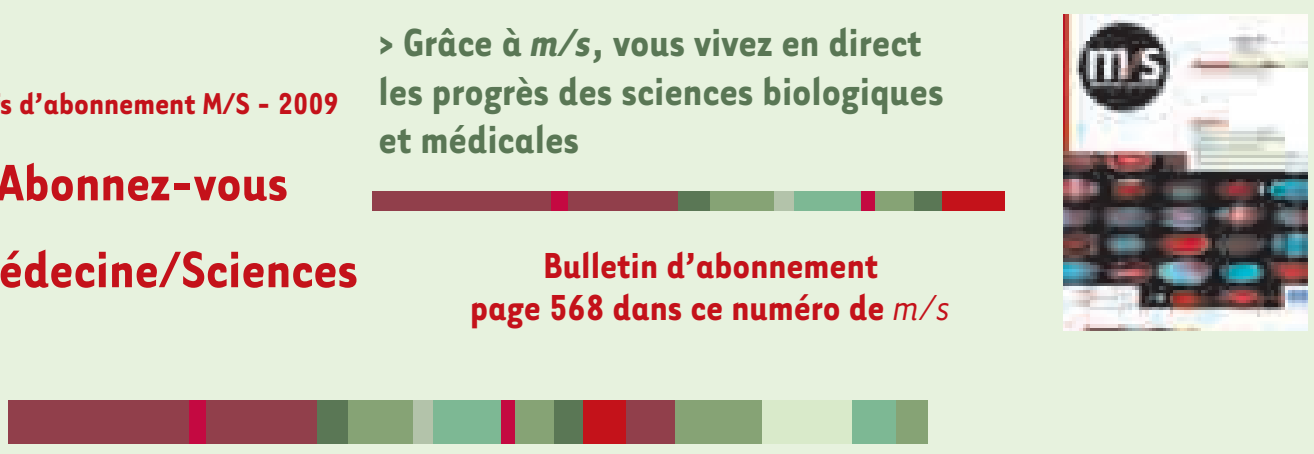